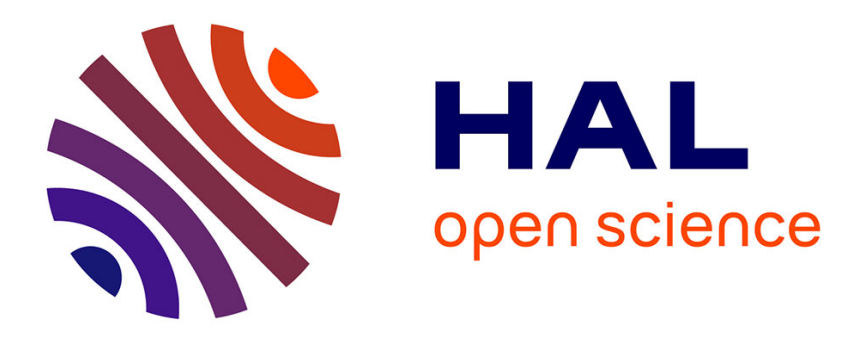

\title{
Les psychanalystes et le transfert de pensée: enjeux historiques et actuels
}

Renaud Evrard, Thomas Rabeyron

\section{To cite this version:}

Renaud Evrard, Thomas Rabeyron. Les psychanalystes et le transfert de pensée: enjeux historiques et actuels. L'Évolution Psychiatrique, 2012, 77 (4), pp.589-598. 10.1016/j.evopsy.2012.05.002 . halshs02137262

\section{HAL Id: halshs-02137262 \\ https://shs.hal.science/halshs-02137262}

Submitted on 22 May 2019

HAL is a multi-disciplinary open access archive for the deposit and dissemination of scientific research documents, whether they are published or not. The documents may come from teaching and research institutions in France or abroad, or from public or private research centers.
L'archive ouverte pluridisciplinaire HAL, est destinée au dépôt et à la diffusion de documents scientifiques de niveau recherche, publiés ou non, émanant des établissements d'enseignement et de recherche français ou étrangers, des laboratoires publics ou privés. 


\section{Les psychanalystes et le transfert de pensée : enjeux historiques et actuels}

Renaud Evrard, Psychologue Clinicien, Doctorant, Université de Rouen Thomas Rabeyron, Psychologue Clinicien, Docteur en Psychologie, ATER, Université Lumière Lyon II

Adresse de correspondance: Evrard Renaud, 45 rue du 11 novembre, 57300 Hagondange. Tel: 0671188186 ; renaud.evrard@etu.univ-rouen.fr

Rabeyron Thomas, 63 chemin de l'Aigas, 69160 Tassin la demi-lune. Tel: 0683140287; trabeyron@wanadoo.fr 


\section{Les rapports complexes de Freud avec le transfert de pensée}

Le rapport des psychanalystes au transfert de pensée peut être décomposé en une palette de positionnements théoriques, techniques et transférentiels. Ainsi, même la position de Freud sera amenée à évoluer. Ses premiers écrits sur le rêve le conduisent à traiter de la signification occulte des rêves, et en particulier du rêve apparemment prémonitoire d'une patiente (Freud, $1899^{1}$ ). Il propose une hypothèse originale, s'appuyant sur les associations et l'histoire de la patiente, expliquant que le souvenir du rêve anticipant l'événement serait en fait une reconstruction après-coup ou paramnésie. Dès cette première hypothèse, il y a la marque d'un abord psychanalytique original qui ne se réduit pas à la question de l'authenticité paranormale du vécu rapporté. A la véridicité se substitue l'importance de la fonction psychologique que prend l'expérience. L'essentiel des positions théoriques de Freud sur la divination dans le rêve paraîtra d'ailleurs plus tardivement dans un article (Freud, 1925) qui aurait dû figurer dans l'édition définitive de L'Interprétation des rêves.

L'intérêt de Freud pour l'occultisme et le transfert de pensée a été grandissant au cours de sa carrière. Six articles de sa part traitent d'occultisme (Freud, 1899, 1919, 1921a, 1921b, 1925, 1932), d'abord pour donner des explications psychanalytiques alternatives aux croyances occultes associées au rêve, jusqu'à développer sa conviction centrée sur le transfert de pensée (Gedankenübertragung). En plus de ces articles, et de quelques autres sur l'interprétation d'un cas de névrose démoniaque (Freud, 1922) ou d'une sensation de déjà-vu (Freud, 1936), ce sont plusieurs œuvres majeures de Freud qui abordent différents aspects de l'occultisme. Dans L'avenir d'une illusion (Freud, 1927), il s'intéresse au spiritisme et aux apparitions, supposant qu'il s'agit de projections à l'extérieur de la réalité psychique inconsciente. Le malaise dans la culture (Freud, 1929) débute sur l'assimilation de l'expérience spirituelle du "sentiment océanique » au fantasme incestueux de retour au sein maternel. Il analysa également, dans L'interprétation des rêves, les apparitions et la divination, concevant cette dernière comme une illusion (Freud, 1900). La pensée superstitieuse fut appréhendée dans Psychopathologie de la vie quotidienne (Freud, 1901) et le crédit accordé par certains aux coïncidences, dont «l'homme aux rats » (Freud, 1909), fut interprété à travers le concept de toute-puissance des pensées, un thème approfondi dans Totem et Tabou (Freud, 1913).

Les investigations de Freud l'ont amené à côtoyer la recherche psychique de son époque, d'où il tire le concept de «télépathie », terme forgé par Frederic Myers de la Society for Psychical Research (SPR) de Londres et souvent donné pour équivalent de «transfert de pensée » (Le Maléfan, 2008). L'importance de ces recherches au tournant du XXe siècle a presque été oubliée aujourd'hui ou confondue avec des mouvements comme le spiritisme (Méheust, 1999 ; Plas, 2000). Le terme d'occultisme, employé par Freud, entraîne d'ailleurs une confusion historique et épistémologique avec le mouvement hétérogène de l'occultisme renaissant au milieu du XIXe siècle (Lachapelle, 2011). En vérité, la pensée de Freud s'inscrivait parmi un ensemble de travaux associant paranormalité et inconscients tels que ceux de Janet, Flournoy, Richet, Grasset, James et Myers.

Dans l'historiographie officielle, l'intérêt du fondateur de la psychanalyse pour la recherche psychique est relativement méconnu. Cette attitude des psychanalystes pourrait n'être que l'écho de la propre ambivalence de Freud. Ainsi, en lisant les textes et les propos épistolaires de ce dernier au sujet de la télépathie, on est frappé par leur rhétorique étonnamment contradictoire (Turnheim, 2008). La question le fascine, mais, en même temps, lui fait peur, et notamment en ce qu'elle vient déstabiliser l'édifice théorique de la

\footnotetext{
Les dates sont celles des premières publications en langue originale.
} 
psychanalyse. D'où une constante tergiversation et des prises de position qui semblent s'opposer, ce qui nécessite une analyse détaillée pour mieux les comprendre (Moreau, 1976).

Ainsi, jusqu'en 1909, Freud affiche un certain scepticisme. Mais à partir de cette date, il change d'opinion. Sous l'influence de Jung et de Ferenczi, qui le convient à certaines expériences avec la voyante berlinoise Frau Seidler, il en vient à accepter l'idée d'un noyau de vérité des faits dits occultes, qui serait constitué par la télépathie. Mais cette évolution n'est pas rendue publique et Freud décourage Ferenczi de publier sur ce sujet. Il a en effet d'autres conseillers, en particulier Jones et Hitschmann, beaucoup plus réticents à l'idée de suivre une psychanalyse qui se fourvoierait avec l'occultisme (Ellenberger, 1970).

Freud devient ensuite membre de la British SPR et de l'American SPR en 1911, et le restera jusqu'en 1938. En 1918, dans sa correspondance avec Fanny Hoppe-Moser, biologiste et parapsychologue suisse fille de la patiente «Emmy von N.», Freud reste prudent mais s'attend à ce que ces recherches ouvrent, dans le meilleur des cas, à un nouveau domaine de faits qui s'intégrera simplement aux autres (Lettre de Freud à Fanny Hoppe-Moser du 10 octobre 1918 ; cf. Bauer, 1986).

Une troisième phase débute en 1921. A partir de cette date, Freud a acquis la certitude de la réalité de la télépathie, au point de s'engager publiquement. Il envoie au parapsychologue Carrington cette déclaration surprenante :

«Si je me trouvais au début de ma carrière scientifique au lieu d'être à sa fin, je ne choisirais peut-être pas d'autre domaine de recherches [que la recherche psychique], en dépit de toutes les difficultés qu'il représente » (Freud, 1966, p. 364).

Puis, à Eitingon, qui vient de lui envoyer le Traité de Métapsychique de Richet (1922), il écrit le 13 novembre 1922 que tout cela «le rendait perplexe jusqu'à lui en faire perdre la tête ». Le 4 février 1922, il confie à ce même Eitingon : " La pensée de cette pomme acide me fait frémir, mais il n'y a pas moyen d'éviter d'avoir à y mordre. » Et c'est encore aux phénomènes paranormaux qu'il doit un de ses plus significatifs actes manqués : lors de la réunion dans la forêt de la Harz, en 1921, il s'aperçoit, au moment de prendre la parole sur la question de l'occultisme, qu'il a oublié le dossier sur lequel devait reposer son exposé...

Dans cet exposé de 1921 et dans son article de 1925, il relate notamment le cas d'une diseuse de bonne aventure qui prédit à sa cliente (la patiente de Freud) qu'elle aurait deux enfants à l'âge de 32 ans. La prophétie ne se réalisa pas mais, explique Freud, il y a eu télépathie car la voyante a perçu le désir inconscient de sa cliente, laquelle voulait imiter sa mère qui dut attendre ses 32 ans pour avoir un enfant. Cette prophétie à moitié-vraie est à l'origine de l'expression d'Octave Mannoni : «Je sais bien, mais quand même » (Sauret, 1982, p. 246). Celle-ci est souvent citée comme la formule de la dénégation, alors qu'elle exprime tout autre chose chez Freud, à savoir le troc d'un «pouvoir de divination» contre une «télépathie soumise à un travail de l'inconscient». Ce pouvoir entendu comme un «don physiologique » sonnera également chez Lacan (1973) comme le mythe d'un «corps plus calé », façon d'interroger la place du transfert de pensée comme objet de la psychanalyse.

Freud optera finalement pour une solution de compromis. Il fait jouer à la télépathie un rôle en marge dans son élaboration théorique, celui de donner du travail de l'inconscient une illustration supplémentaire. Freud se fait fort d'introduire dans la raison une subversion nouvelle, tout en protégeant l'ordre des choses. L'ambiguïté de ce compromis entraîne cependant fréquemment sa remise en question. Rosolato conteste ainsi l'interprétation de Freud en termes de télépathie de certaines concordances, en percevant chez lui un aveuglement par son désir d'être devin, d'être «au moins l'égal d'Édipe pour résoudre les énigmes » (Rosolato, 1976, p. 23). Plé (1968) analyse également cet attrait de Freud comme un déplacement de son angoisse existentielle. Quant à Roustang (1980, p. 75), il propose une 
explication de la télépathie par la rencontre de chaînes signifiantes, pointant alors ce qu'il nomme «la psychose de Freud».

\section{Transfert de pensées et pensées de transfert entre Freud, Ferenczi et Jung}

Ferenczi (Fodor, 1979; Lorin, 1989) et Jung (Jaffé, 1960 ; Fodor, 1972) sont deux autres psychanalystes dont l'intérêt pour le transfert de pensée est bien documenté. Ferenczi s'était imprégné très jeune des ouvrages sur les sciences psychiques. Sa première publication (Ferenczi, 1899), dans une revue médicale hongroise, fut consacrée au spiritisme. Le choix de la revue lui fut même dicté lors d'une crise d'écriture automatique (Fodor, 1979) !

On connaît la grande curiosité de Ferenczi pour ce domaine surtout à travers ses correspondances et son journal clinique. Ainsi, il écrivait à Freud le 22 novembre 1910 qu'il pensait être un «formidable voyant» car il lisait dans ses associations libres les pensées de ses patients. Selon lui, la future méthodologie analytique devrait en tirer profit. Freud lui répondra le 3 décembre 1910 en lui demandant d'accumuler les données avant de se prononcer publiquement. Bien qu'il lui conseillait la prudence, Freud confia à Jung (lettre du 15 juin 1911) qu'il était très impressionné : les expériences de Ferenczi l'ont « rendu humble et brisé ses présomptions à l'encontre de l'occultisme ». Un peu plus tard (le 10 janvier 1912), toujours dans sa correspondance avec Jung, il considère que les preuves de Ferenczi à l'appui de la transmission de pensée sont une des deux contributions à la psychanalyse les plus importantes des derniers temps. Ce faisant, Freud comblait certaines des attentes de Jung, tout en faisant de Ferenczi le fils prodigue.

D'autres psychanalystes n'ont pas hésité à franchir le pas qui retenait Freud. Jung en est un exemple. Après avoir rédigé sa thèse sur la psychologie et la psychopathologie des phénomènes dits occultes (Jung, 1902), il poursuivra quelques travaux connexes et apportera son soutien aux sciences psychiques. Il collaborera aux congrès internationaux et assistera aux expériences de matérialisations ectoplasmiques du baron von Schrenk-Notzing en compagnie de Bleuler. Sa correspondance avec le physicien Wolfgang Pauli fut à la base de son modèle de la synchronicité pour expliquer les «coïncidences significatives » (Jung \& Pauli, 1952).

Freud était donc pressé par deux de ses plus proches disciples pour qu'il s'occupe d'occultisme. Gori (1996) a commenté cette situation en lui donnant un éclairage original. Partant de l'idée qu'une théorie ne s'élabore que dans le transfert, son évaluation passe par l'exploration du transfert au maitre dont elle est contemporaine. En ce sens, il analyse les intérêts de Ferenczi et Jung pour le transfert de pensée comme des acting out adressés à Freud. Jung et Ferenczi semblent effectivement se servir de l'occultisme pour venir «chatouiller» Freud, pour exprimer la part négative de leur transfert, pour critiquer un dogmatisme psychanalytique qui, dans le même temps, les fascine. En l'absence d'un véritable cadre didactique, on passe des pensées de transfert au transfert de pensée comme recours pour élaborer l'inachevé de la relation analytique (Gori, 1996, p. 57) tout en abordant la question de la suggestion dans la cure (Roustang, 1980). Pour Gori (1996, p. 61), plusieurs théorisations pertinentes ont découlé de l'analyse de ces débats: par exemple, la compréhension du contre-transfert et l'intérêt pour l'empiétement traumatique de l'environnement, très développés chez Ferenczi ; ainsi que la notion de complaisance au hasard encore peu discutée.

Toutefois, Gori minimise l'intérêt personnel de Freud pour la télépathie. On peut également remarquer que plus d'une dizaine de chercheurs, psychanalystes ou autres, sont venus solliciter Freud pour qu'il participe plus activement aux sciences psychiques (Moreau, 1976), sans qu'on puisse pour autant attribuer ces appels à une relation transférentielle de disciple à maître. A plusieurs reprises (par exemple, Stekel, 1921 ; Deutsch, 1926), ce sont les publications audacieuses de psychanalystes sur ces sujets qui ont frayé la voie à Freud. Ainsi, 
sans négliger cette part de transfert à Freud dans le rapport à la télépathie, il semble important d'explorer plus largement cet intérêt des premiers psychanalystes.

\section{Positionnements et théorisations des premiers analystes}

Les historiens de la psychologie tendent à reconnaître que le transfert de pensée fut l'un des premiers objets d'étude de la psychologie naissante au tournant du XXe siècle (Méheust, 1999 ; Plas, 2000). Les premiers psychologues ont ainsi :

... presque tous été, de près ou de loin, impliqués dans des recherches qui seraient, de nos jours, exclues du champ de la psychologie et renvoyées à la parapsychologie comme, par exemple, l'étude de ces phénomènes que l'on appelle actuellement " perception extra-sensorielle " ou " télépathie ".2

Celle-ci se prêtait particulièrement bien à l'expérimentation scientifique, avec en arrière-plan cette volonté d'isoler des capacités subconscientes hors du commun dont seraient doués en particulier des sujets hystériques. Les psychanalystes ne purent échapper à cet engouement et ont métabolisé à leur façon cet héritage. Ainsi, Wilhem Stekel (1921) publia une monographie sur le rêve télépathique chez des patients névrotiques qui mettaient en évidence que ce motif jaillissait dans des situations émotionnelles fortes, en particulier l'amour, la jalousie et l'anxiété. Il manqua néanmoins de percevoir le lien entre ces rêves télépathiques et les processus de distorsion connus de la psychanalyse, ce qui revint à Freud (1921b). Toutefois, Freud disait ne pas disposer de «rêves télépathiques » ayant émergé lors d'une cure et se référa donc au témoignage d'un correspondant. La présence de ce «travail du rêve » faisait dire à Freud que la psychanalyse avait un rôle à jouer pour faire émerger un contenu télépathique passé inaperçu.

Ce rapprochement généralisé entre rêve et télépathie ouvrait la porte à un rencontre du matériel des analystes et des sociétés de recherche psychique (Róheim, 1932 ; Zulliger, 1934 ; Fodor, 1942; Pederson-Krag, 1947) et plus tard à des expérimentations sur le rêve télépathique en laboratoire (Ullman, Krippner, \& Vaughan, 1977). Fodor (1942) tenta de montrer que certains rêves d'analysants sont mieux compris en faisant appel à des événements qui ne sont pas contenus dans leurs seules associations, par exemple les propres rêves de l'analyste en relation avec son analysant. Hélène Deutsch (1926) et Dorothy Burlingham (1935) firent également l'étude des «processus occultes » en cours d'analyse d'adultes et d'enfants.

Le hongrois Hollós (1933), élève de Ferenczi devenu président de l'association psychanalytique de Hongrie l'année même de cet article, proposera également des observations d'événements télépathiques sur plusieurs années. Ceux-ci provenaient à la fois du patient et de l'analyste, si bien que le meilleur parti fut pour lui de considérer que ce matériel est à l'intersection de forces prenant leur source dans les deux inconscients à la fois. Cette vision dynamique trouve d'ailleurs un écho dans la formulation de la «chimère » de De M'Uzan (1977, 1994).

Contrairement à l'abord de ces auteurs, plusieurs psychanalystes tentèrent de dissuader Freud de mêler l'occultisme à la psychanalyse. Hitschmann (1910, 1923) développa par exemple des explications psychologiques en termes de faux souvenirs, de fraudes délibérées, de «prophéties» prenant leur source dans un désir et une anticipation rationnelle, d'embellissement narratif, d'imagerie stimulée par un complexe affectif et dont l'étrangeté provient de sa distorsion par un travail inconscient. Le psychisme humain montre toute sa complexité dans ces expériences qui, au même titre que les rêves, les lapsus et les actes manqués, révèlent l'œuvre d'un fonctionnement psychique inconscient.

\footnotetext{
2 Plas, 2000, p. 16.
} 


\section{Controverses autour de la réalité du transfert de pensée}

En 1925, Freud terminait son article consacré à la signification occulte des rêves par une sorte d'encouragement adressé aux analystes : "Ce serait très satisfaisant si, à l'aide de la psychanalyse, nous pouvions authentifier la télépathie et en avoir une connaissance plus approfondie ». Plusieurs analystes (en particulier Servadio, Von Winterstein, Fodor, Vinchon, Ehrenwald, Eisenbud, Perderson-Krag et Ullman) répondirent à cet encouragement en s'appuyant à la fois sur la psychanalyse et les recherches psychiques. Des controverses jaillirent en écho à leurs nombreuses publications. La parution en 1953 de l'anthologie de textes réunis par George Devereux, mettant fin à la dispersion des contributions, marque l'apogée de ce débat.

Cette anthologie accueillait une controverse très marquante pour le découpage $\mathrm{du}$ champ psychothérapeutique américain, avec une critique des données de Pederson-Krag, Eisenbud et Fodor par Albert Ellis. Formé à la psychanalyse, Ellis quittera ce champ, notamment suite à cette controverse, pour fonder sa propre école de «thérapie rationnelleémotive », berceau des thérapies cognitivo-comportementales. L'un de ses buts était de modifier les croyances irrationnelles du patient en lui démontrant qu'elles sont les sources de ses souffrances émotionnelles. Le principal exemple sur lequel il étayait sa thèse était la correction des perceptions supposées télépathiques en analyse. Celles-ci lui servirent surtout de repoussoirs, lui assurant le titre de «prince de la raison » qui lui sera décerné plus tard.

Cette controverse est particulièrement riche d'enseignements car elle va permettre aux psychanalystes de définir plus précisément quelle attitude clinique tenir face à des patients qui pensent vivre des phénomènes télépathiques. L'argumentation d'Ellis consiste à ramener tous les exemples de télépathie relatés par des psychanalystes à de simples coïncidences. Avec la loi des grands nombres, même l'improbable peut se produire et la quantité de matériel que croise un analyste aide à faire survenir l'improbable. En plus de cette critique ramenant les concordances à des coïncidences dues au hasard, Ellis avançait que les éventuelles causes de ces coïncidences seraient plutôt à chercher dans la suggestion imprimée par l'analyste que dans la télépathie.

Ellis proposait également une liste de recommandations pour les psychanalystes qui voudraient prouver la télépathie. Or, la plupart d'entre elles ne sont pas réalisables dans un contexte analytique. Éliminer tout facteur affectif, toute suggestion, toute croyance antérieure, demander à ce que les motifs oniriques ne se présentent pas sous une forme symbolique, de sorte que la télépathie émerge dans un vide absolu et stérilisé, ce n'est possible que dans un laboratoire de psychologie expérimentale. Devereux répondra qu'il n'y a pas de phénomènes qui existeraient en soi, ni du côté de la parapsychologie, ni du côté de la psychanalyse, ni ailleurs. Ces phénomènes existent par rapport à un cadre qui les formalisent, ce qui rend possible un relativisme et donc un accès psychanalytique à ces phénomènes.

La controverse avec Ellis a incité Devereux à discuter sur le plan technique des possibilités pour l'analyste de travailler avec les occurrences de transfert de pensée se produisant dans la situation analytique (Devereux, 1953, p. 391-417). Son modèle est un des plus aboutis sur le plan de la neutralité. Celle-ci est heuristique : si l'analyste ne peut affirmer a priori que deux événements sont reliés de façon paranormale, il peut aider à les relier de façon analytique. Ainsi, qu'il y ait du matériel «hétéropsychique » ou «intrapsychique » (Ehrenwald, 1947), il y aura de toute manière des répercussions inconscientes qu'il est important d'analyser (Devereux, 1953, p. 43). Cette réflexion se poursuivra dans ses futurs ouvrages où le contre-transfert prendra progressivement une place centrale dans l'épistémologie et la pratique des sciences du comportement (Devereux, 1967). 


\section{Actualité du transfert de pensée dans la clinique contemporaine}

Par la suite, aucune position officielle ne venant lever l'ambiguïté freudienne au sujet du transfert de pensée, les positions les plus diverses ont été tenues par des psychanalystes (mais aussi par des non-psychanalystes, comme Derrida, 1983). Plusieurs ont proposé du matériel supplémentaire s'ajoutant à celui apporté par Freud (Eschel, 2006 ; Bendit, 1951 ; Totton, 2003 ; Chaperot, 2011), d'autres ont cherché à commenter le matériel de départ pour développer des interprétations complexes des processus en jeu (Granoff et Rey, 1983/2005; Barbier \& Decourt, 1998 ; Bernat, 2001), d'autres ont commenté et même contribué aux recherches psychiques (Mayer, 2007 ; Eisenbud, 1966 ; Ullman, Krippner, Vaughan, 1977). La plupart de ces contributions restent isolées les unes des autres et ce manque d'intégration fait du savoir psychanalytique sur l'occulte un objet toujours recommencé. Il nous semble ainsi que les enjeux majeurs du transfert de pensée, tels qu'ils ont été développés par Freud sur les plans cliniques et théoriques, n'ont pas encore trouvé l'écho qu'ils méritaient.

Pourtant, la question du transfert de pensée se retrouve au croisement d'un certain nombre de courants psychanalytiques tentant actuellement de penser les spécificités des relations intersubjectives. C'est tout d'abord autour de la question de la transmission psychique inconsciente, notamment dans la lignée des travaux de Mélanie Klein, que s'organisent des réflexions proches de celles des premiers psychanalystes (Ciccone, 1999). Elles interrogent plus largement les différentes modalités de transmission, notamment en lien avec les transmissions psychiques trans-générationnelles (Kaës et al., 1993).

La transmission de pensée se trouve également parmi les préoccupations des théoriciens des frontières du moi au carrefour de l'individuel et du groupal. Anzieu (2003), avec le concept d' « enveloppe psychique », ou Bion (1962) et la notion de «membrane psychique », proposent une réflexion sur la perméabilité aux contenus psychiques d'autrui. Ces notions d'enveloppe et de perméabilité se retrouvent également au premier plan dans les dynamiques groupales. Celles-ci impliquent des processus archaïques qui font resurgir des modalités intersubjectives précoces invoquant la question du transfert de pensée (Moreno, 1923). Ce lien avec l'archaïque se retrouve d'ailleurs également dans la clinique des bébés (FisherBernardino, 2005). Dans ces différents cas, le transfert de pensée se trouve ainsi interrogé comme l'une des modalités potentielles de communication et d'échange aux niveaux intersubjectifs et intrapsychiques.

Un autre axe concerne plus spécifiquement l'intersubjectivité en cours d'analyse. Les réflexions de De M'Uzan autour du concept de «chimère », auxquelles nous avons déjà fait allusion, approfondissent celles de Freud et Ferenczi sur ce point. De M'Uzan (1994) affirme que la situation analytique est propice à l'émergence de contenus apparemment hétéropsychiques, des productions étranges et spontanées d'une précision parfois étonnante. De ce point de vue, le transfert de pensée se trouve abordé de biais, sans traiter directement de son aspect potentiellement «objectif»- le plus controversé -, dans ce qui se transfert plus spécifiquement entre un patient et son analyste, dans un cadre favorisant des phénomènes de régression.

Mais c'est peut-être de la psychologie des expériences exceptionnelles, ou vécues comme «paranormales», que proviennent les apports les plus originaux. Nous pouvons notamment remarquer les travaux récents de Pascal Le Maléfan (2008) portant sur les hallucinations télépathiques ou véridiques, les expériences de mort imminente (Le Maléfan, 2001) et les sorties hors du corps (Le Maléfan, 2005). Mais aussi les recherches d'Anne Juranville (2001) sur la possession ou de Jean-Claude Maleval et Nathalie Charraud (1997) sur le syndrome d'enlèvement par des extra-terrestres. Des auteurs comme Djohar Si Ahmed (2006) ou Elizabeth Laborde-Nottale (1990) ont traité plus spécifiquement du transfert de pensée à travers diverses pratiques cliniques. Nous avons également récemment proposé une ré-élaboration de ces expériences exceptionnelles à partir de différentes cliniques, notamment 
celle des adolescents (Evrard, 2010), et d'un rapprochement avec les neurosciences (Rabeyron, 2009 ; Rabeyron, Chouvier, \& Le Maléfan, 2010). Les expériences exceptionnelles ont pour point commun d'impliquer fréquemment des formes de communication qui réactivent l'idée d'un transfert de pensée. Cette phénoménologie spontanée souvent teintée d'inquiétante étrangeté représente un matériel clinique original pouvant mener à des hypothèses novatrices dans la continuité des premières réflexions de Freud.

\section{Conclusion}

Comme nous l'avons vu, l'attitude de Freud à l'égard du transfert de pensée à évolué au cours de sa vie, la rendant complexe et parfois ambiguë. Influencé par la recherche psychique de son époque, il a tenté d'extraire de l'occultisme la télépathie comme noyau de faits réels. Pour des raisons politiques et épistémologiques, cet objet fut cependant longtemps situé à la fois à l'intérieur et à l'extérieur du territoire psychanalytique, suscitant de larges débats dans les années 1950.

Regroupées, ces contributions de psychanalystes montrent que le «transfert de pensée » reste un lieu important de questionnement de l'épistémologie freudienne, tout particulièrement dans les théorisations du transfert et du contre-transfert. Il est à la fois à la source et au carrefour de diverses réflexions cliniques contemporaines qui, en retour, conduisent à repenser les modalités de la transmission psychique. En étant davantage en phase avec les préoccupations initiales de Freud, ces cliniques supportent des questionnements fondamentaux : quels sont les rapports entre suggestion et transfert ? Le transfert de pensée est-il une modalité intersubjective spécifique, et si oui, de quelle nature ? Comment une clinique psychanalytique peut-elle accueillir les expériences spontanées de télépathie ? Ces questions sont fondamentales dans la mesure où elles interrogent plus largement, comme le souligne Bernat, la façon dont « une perception accède ou non à la conscience » (Bernat, 2001, p. 15). Le transfert de pensée apparaît ainsi comme un objet en marge qui pourrait participer plus avant d'une compréhension globale de la réalité psychique.

\section{Bibliographie}

ANZIEU, D. 2003. Les enveloppes psychiques, Paris, Dunod.

BARBIER, A.; DECOURT, P. 1998. Transmission, transfert de pensée, interprétation, 13èmes journées Occitanes de Psychanalyse (1997), Montpellier, Editions du Monde interne.

BAUER, E. 1986. “ Ein noch nicht publizierter Brief Sigmund Freuds an Fanny Moser über Okkultismus und Mesmerismus ”, dans Freiburger Universitätsblätter, 25, 93-110.

BENDIT, L.J. 1951. La connaissance paranormale et sa place dans la psychologie humaine, Paris, L'Arche.

BERNAT, J. 2001. Transfert et pensée, Paris, L'esprit du temps.

BION, W.R. 1962. Aux sources de l'expérience, Paris, PUF, 2003.

BURLINGHAM, D.T. 1935. "Child analysis and the mother ”, dans Psychoanalytic Quarterly, 4, 69-92.

CHAPEROT, C. Phénomènes d'allure télépathique dans la relation avec des patients schizophrènes : hypothèse d'une potentielle nocivité de la pensée soignante. L'Evolution psychiatrique, 2011, 76(2): 273-286. 
CICCONE, A. 1999. La transmission psychique inconsciente: identification projective et fantasme de transmission, Paris, Dunod.

DE M'UZAN, M. 1977. De l'art à la mort, Paris, Gallimard.

DE M'UZAN, M. 1994. La bouche de l'inconscient, Paris, Gallimard.

Derrida, J. (1983). Télépathie. Cahiers Confrontation, n¹0, 201-230.

DEUTSCH, H. 1926. “Processus occultes pendant l'analyse ”, dans Cas cliniques et autoanalyse 1918-1930, Paris, Le Seuil, 1992, 85-101.

DEVEREUX, G. 1953. Psychoanalysis and the occult, New York, International University Press.

DEVEREUX, G. 1967. De l'angoisse à la méthode dans les sciences du comportement, Paris, Flammarion, 1980.

EHRENWALD, J. 1947. Telepathy and Medical Psychology, London, Allend \& Unwin.

EISENBUD, J. 1966. The World of Ted Serios: "Thoughtographic" Studies of an Extraordinary Mind, New York, William Morrow.

Ellenberger, H.F. (1970/1994). A la découverte de l'inconscient : histoire de la psychiatrie dynamique. Paris : Fayard.

ESCHEL, O. 2006. “Where are you, my beloved ? On absence, loss, and the enigma of telepathic dreams ”, dans International Journal of Psychoanalysis, 87, 1603-1627.

EVRARD, R. 2010. “ Psychiser le Maître absolu : solutions pubertaires par le paranormal ”, Adolescence, 74, 841-854.

FERENCZI, S. 1899. “ Le spiritisme”, dans Les Ecrits de Budapest, Paris, Epel, 1994.

FISCHER BERNARDINO, M. 2005. “ Un retour à Freud pour fonder la clinique psychanalytique des bébés et de leurs parents : les études sur la télépathie ", dans Figures de la psychanalyse, 11, 207-213.

FODOR, N. 1942. “Telepathic Dreams ”, dans American Imago, 3, 61-87.

FODOR, N. 1972. Freud, Jung, and Occultism, New York, New York University Book.

FODOR, N. 1979. “ Les aventures psychiques de Sándor Ferenczi ”, dans Le Coq-Héron, 73, 20-32.

FREUD, S. 1899. “Une prémonition onirique accomplie ”, dans Résultats, idées, problèmes I, Paris, PUF, 1994, 109-111.

FREUD, S. 1900. L'interprétation des rêves, Paris, PUF, 1999.

FREUD, S. 1901. Psychopathologie de la vie quotidienne, Paris, Petite Bibliothèque Payot, 1997.

FREUD, S. 1909. “ Remarques sur un cas de névrose obsessionnelle (L'homme aux rats) ", dans Cinq psychanalyses, Paris, PUF, 1954, 199-261.

FREUD, S. 1913. Totem et tabou, Paris, Gallimard, 1993.

FREUD, S. 1919. “L'inquiétante étrangeté ”, dans Essais de psychanalyse appliquée, Paris, Gallimard, 1971, 163-210.

FREUD, S. 1921a. " Psychanalyse et télépathie ”, dans Euvres complètes, XVI, Paris, PUF, 1991, 10-15. 
FREUD, S. 1921b. “Rêve et télépathie ”, dans Euvres complètes, XVI, Paris, PUF, 1991, 119-144.

FREUD, S. 1922. “Une névrose démoniaque au XVIIe siècle ”, dans Essais de psychanalyse appliquée, Paris, Gallimard, 1971, 211-251.

FREUD, S. 1923. "Remarques sur la théorie et la pratique de l'interprétation des rêves ", dans Résultats, idées, problèmes II, Paris, PUF, 1985, 79-91.

FREUD, S. 1925. “Quelques suppléments à l'interprétation des rêves ”, dans CEuvres complètes, XVII, Paris, PUF, 1992, 185-188.

FREUD, S. 1927. L'avenir d'une illusion, Paris, PUF, 1971.

FREUD, S. 1929. Le malaise dans la culture, Paris, PUF, 2004.

FREUD, S. 1932. "Rêve et occultisme ”, dans Nouvelles conférences d'introduction à la psychanalyse, Paris, Gallimard, 1984, 45-79.

FREUD, S. 1936. “ Un trouble de mémoire sur l'Acropole. Lettre à Romain Rolland ”, dans Résultats, idées, problèmes II, Paris, PUF, 1985, 221-230.

FREUD, S. 1966. Correspondance, 1873-1939, Paris, Gallimard.

GORI, R. 1996. “Pensées de transfert ou transfert de pensée ”, dans La preuve par la parole : Sur la causalité en psychanalyse, Paris, PUF, 51-73.

GRANOFF, W.; REY, J.-M. 1983. L'occulte : objet de la pensée freudienne, Paris, PUF. [réédition $2005:$ La transmission de pensée]

HITSCHMANN, E. 1910. “ A critique of clairvoyance ”, dans Wiener Klinische Rundschau, 24, 94-95.

HITSCHMANN, E. 1923. “Telepathie und Psychoanalyse”, dans Imago, 9, 368-382.

HOLLÓS, I. 1933. " Psychopathologie alltäglicher telepathischer Erscheinungen ”, dans Imago, $19,529-546$.

JAFFE, A. 1960. “ C.G. Jung und die Parapsychologie ”, dans Zeitschrift für Parapsychologie und Grenzgebiete der Psychologie, 4, 8-23.

JUNG, C.G. 1902. " Sur la psychologie et la psychopathologie des phénomènes dits occultes ", dans L'énergétique psychique, Zurich, Librairie de l’Université, 1956, 118-218.

JUNG, C.G., \& PAULI, W. 1952. Naturerklärung und Psyche, Zürich; Rascher Verlag.

JURANVILLE, A. 2001. Figures de la possession: Actualité psychanalytique du démoniaque, Grenoble, Presses Universitaires de Grenoble.

KAËS, R., FAIMBERG, H., ENRIQUEZ, M., BARANES, J.J. 1993. Transmission de la vie psychique entre générations, Paris, Dunod.

LABORDE-NOTTALE, E. 1990. La voyance et l'inconscient, Paris, Seuil.

LACAN, J. 1973. Les non-dupes errent, Séminaire inédit, leçon du 20 novembre.

LACHAPELLE, S. 2002. A world outside science: french attitudes toward mediumistic phenomena, 1853-1931, Thèse d'histoire à l'Université Notre-Dame, Indiana, Etats-Unis.

LE MALEFAN, P. 2001. “Les expériences de mort imminente (EMI) au regard de la clinique psychanalytique ", dans Perspectives Psy, 40, 125-132.

LE MALEFAN, P. 2005. “ La «sortie hors du corps » est-elle pensable par nos modèles cliniques et psychopathologiques? Essai de clinique d'une marge. A propos d'un cas ", dans L'Evolution psychiatrique, 70, 513-534. 
LE MALEFAN, P. 2008. “ L'hallucination télépathique ou véridique dans la psychopathologie de la fin du XIXe siècle et du début du XXe siècle ”, dans L'Evolution Psychiatrique, 73, $15-40$.

LORIN, C. 1989. “ Sandor et les fantômes ”, dans Nouvelle Revue d'Ethnopsychiatrie, 13, 229-236.

MALEVAL, J.C., \& CHARRAUD, N. 1997. “ Modernité du démoniaque ”, dans Psychologie clinique, 4, 117-130.

MAYER, E.L. 2007. Extraordinary Knowing: Science, Skepticism, and the Inexplicable powers of the Human Mind, New York, Bantam Dell.

MEHEUST, B. 1999. Somnambulisme et médiumnité (2 tomes), Paris, Les Empêcheurs de Penser en Rond.

MOREAU, C. 1976. Freud et l'occultisme, Paris, Privat.

MORENO, J. 1923. Le théâtre de la spontanéité, Paris, Desclée de Brouwer, 1984.

PEDERSON-KRAG, G. 1947. “ Telepathy and repression ”, dans Psychoanalytical Quarterly, 21, 607-659.

PLAS, R. 2000. Naissance d'une science humaine, la psychologie: les psychologues et le « merveilleux psychique », Rennes, PUR.

PLE, A. 1968. Freud et la religion, Paris, Editions du Cerf.

RABEYRON, T. 2009. " Les expériences exceptionnelles : entre neurosciences et psychanalyse ", dans Recherches en Psychanalyse, 8.

Rabeyron, T., Chouvier, B., Le Maléfan, P. (2010). Clinique des expériences exceptionnelles : du trauma à la solution paranormale. L'Evolution Psychiatrique, 75(4), 633-653.

RICHET, C. 1922. Traité de métapsychique, Paris, Felix Alcan.

RÓHEIM, G. 1932. “Telepathy in a dream ”, dans The Psychoanalytic Quarterly, 1, 227-291.

ROSOLATO, G. 1976. "La scission que porte l'incroyable", dans Nouvelle Revue de Psychanalyse, 18, 16-23.

ROUSTANG, F. 1980. ...Elle ne le lâche plus, Paris, Les éditions de minuit.

SAURET, M.-J. 1982. Croire ? Approche psychanalytique de la croyance, Toulouse, Privat.

SI AHMED, D. 2006. Comment penser le paranormal, Paris, L'Harmattan.

STEKEL, W. 1921. Der telepathische Traum, Berlin, Johannes Baum.

TOTTON, N. 2003. Psychoanalysis and the Paranormal: Lands of Darkness, London, New York: Karnac.

TURNHEIM, M. 2008. "Freud le médium (Notes sur l'affaire de la télépathie) ”, dans Psychanalyse, 12, 41-53.

ULLMAN, M., KRIPPNER, S., \& VAUGHAN, A. 1977. La télépathie par le rêve, Paris, Tchou.

ZULLIGER, H. 1934. " Prophetic dreams ", dans International Journal of Psychoanalysis, 15, 191-208. 


\section{Les psychanalystes et le transfert de pensée : enjeux historiques et actuels}

\section{Résumé}

Le concept de "transfert de pensée » a une position particulière au sein $d u$ champ psychanalytique. Il est tout d'abord un objet en marge car il tire son origine des sciences psychiques de la fin du XIXe siècle. C'est aussi un objet extrait par la psychanalyse aux ténèbres de l'occultisme, Freud considérant le transfert de pensée comme en étant le noyau véridique. Mais c'est aussi un corps étranger car même s'il a été appréhendé par différents auteurs, il demeure mal intégré au corpus psychanalytique. Nous essaierons de montrer comment ce concept a tout d'abord joué un rôle heuristique vis-à-vis de la théorisation du transfert et du contre-transfert. Nous verrons ensuite comment il fut repris par les générations successives d'analystes ainsi que les vifs débats qu'il engendra. Enfin, nous dégagerons la pertinence de ce concept au regard d'enjeux cliniques et théoriques actuels.

Mots-clefs : transfert de pensée - contre-transfert - occultisme - histoire de la psychanalyse - expériences exceptionnelles

\section{Psychoanalysts and thought transference: historical and current issues}

\section{Abstract}

The concept of "thought transference" has a special position within the psychoanalytic field. It is first an object on the sidelines because it originated in the psychical sciences of the late nineteenth century. It is also an object extracted by psychoanalysis to the darkness of the occultism, Freud considering the thought transference as being its true nucleus. But it is also a foreign body because even if it was apprehended by different authors, it remains poorly integrated into the psychoanalytic corpus. We try to show how this concept had first played a heuristic role vis-à-vis the theorizing of transference and countertransference. We then see how it was taken over by successive generations of analysts as well as heated debate it engendered. Finally, we will bring out the relevance of this concept in terms of clinical and current theoretical issues.

Key-words: thought transference - countertransference - occultism - history of psychoanalysis - exceptional experiences 